\title{
ON ISOMETRIES OF EUCLIDEAN SPACES
}

F. S. BECKMAN AND D. A. QUARLES, JR.

1. Introduction. A transformation of a Euclidean line onto itself which preserves unit distances is not necessarily an isometry. This is shown by the simple example of the transformation which displaces all integral points one unit in the same direction and leaves all other points fixed.

On the other hand, we show in this paper that a transformation of Euclidean $n$-space $E^{n}(2 \leqq n<\infty)$ which preserves a single nonzero length must be a Euclidean (rigid) motion of $E^{n}$ onto $E^{n}$. It is an immediate consequence that the result also holds for all finite-dimensional unitary spaces. For, if it were false for a unitary space of dimension $n$, then it would be false for a Euclidean space of dimension $2 n$.

That this result fails for $E^{\infty}$, the (real) Hilbert space of infinite sequences of real numbers $\{X\}=\left\{\left(x_{1}, x_{2}, \cdots\right)\right\}$ where $\sum_{i=1}^{\infty} x_{i}^{2}$ exists, is shown by the following example.

There is in $E^{\infty}$ a denumerable and everywhere dense set of points. Let such a set be denoted by $\left\{Y_{i}\right\}$. Define a transformation, $R$, of $E^{\infty}$ into $\left\{Y_{i}\right\}$ in such a way that the distance $d(X, R X)<1 / 2$. Also, define a transformation, $S$, on $\left\{Y_{i}\right\}$ so that $S Y_{i}=A_{i}$ where $A_{i}$ $=\left(a_{i 1}, a_{i 2}, \cdots\right)$ and $a_{i j}=\delta_{i j} 2^{1 / 2} / 2\left(\delta_{i j}\right.$ is the Kronecker delta). It may be readily seen that $T=S R$ is a transformation of $E^{\infty}$ into itself which preserves the unit distance. For, if $d\left(X_{1}, X_{2}\right)=1$, then $R X_{1} \neq R X_{2}$, $T X_{1} \neq T X_{2}$, and therefore $d\left(T X_{1}, T X_{2}\right)=1$. However, not all distances are preserved. For, if $X_{1}$ and $X_{2}$ are any two points in $E^{\infty}$, then $d\left(T X_{1}, T X_{2}\right)$ is either 0 or 1 .

\section{Principal result.}

THEOREM. Let $T$ be a transformation (possibly many-valued) of $E^{n}$ $(2 \leqq n<\infty)$ into itself. Let $d(p, q)$ be the distance between points $p$ and $q$ of $E^{n}$, and let $T p, T q$ be any images of $p$ and $q$, respectively. If there is a length $a>0$ such that $d(T p, T q)=a$ whenever $d(p, q)=a$, then $T$ is a Euclidean transformation of $E^{n}$ onto itself.

REMARK. By a change of scale we can assume in the proof that $a=1$.

3. Proof of theorem for $n=2$.

Received by the editors December 3, 1952 and, in revised form, February 21, 1953. 
Lemma 1. $T$ is single-valued.

Proof. If $T$ were not single-valued, there would be at least one point, say $p_{1}$, with at least two images, say $T p_{1}^{(1)}$ and $T p_{1}^{(2)}$. Consider two points $p_{2}$ and $p_{3}$ which together with $p_{1}$ comprise the vertices of an equilateral triangle with unit side. Since this kind of triangular relationship must be preserved for each set of images of $p_{1}, p_{2}$, and $p_{3}$, it follows that $T p_{1}^{(1)}$ and $T p_{1}^{(2)}$, together with an image $T p_{2}$ of $p_{2}$ and an image $T p_{3}$ of $p_{3}$, comprise the vertices of a rhombus of unit side with $d\left(T p_{1}^{(1)}, T p_{1}^{(2)}\right)=3^{1 / 2}$. Now consider two rhombi, each congruent to the previously mentioned, whose vertices are $p_{1}, p_{2}, p_{4}$, $p_{3}$ and $p_{1}, p_{5}, p_{7}, p_{6}$ respectively, and where $d\left(p_{1}, p_{4}\right)=d\left(p_{1}, p_{7}\right)=3^{1 / 2}$ while $d\left(p_{4}, p_{7}\right)=1$. Due to the relationship between $p_{2}, p_{3}$, and $p_{4}$, there must be an image, say $T p_{4}$, of $p_{4}$ which coincides with either $T p_{1}^{(1)}$ or $T p_{1}^{(2)}$. Since images, say $T p_{6}$ and $T p_{6}$, of $p_{5}$ and $p_{6}$ must be at a unit distance from each other and from both $T p_{1}^{(1)}$ and $T p_{1}^{(2)}$, it follows that the set $\left\{T p_{5}, T p_{6}\right\}$ must coincide with the set $\left\{T p_{2}, T p_{3}\right\}$. Thus, due to the relationship between $p_{6}, p_{6}$, and $p_{7}$, there must be an image, say $T p_{7}$, of $p_{7}$ which coincides with either $T p_{1}^{(1)}$ or $T p_{1}^{(2)}$, implying that $d\left(T p_{4}, T p_{7}\right)=0$ or $3^{1 / 2}$. The contradiction that $d\left(p_{4}, p_{7}\right)$ $=1$ is not preserved by $T$ establishes that $T$ is single-valued, i.e. $d=0$ is preserved.

LEмma 2. If $3^{1 / 2}-1 \leqq d\left(p_{1}, p_{2}\right) \leqq 3^{1 / 2}+1$, then $T p_{1} \neq T p_{2}$.

Proof. It is clear that the vertices of an equilateral triangle with unit side are transformed onto the vertices of a congruent triangle, and that a unit circle is transformed into a congruent circle.

Consider the unit circles $C_{1}$ and $C_{2}$ with centers at $p_{1}$ and $p_{2}$. It is possible to construct an equilateral triangle of unit side with two vertices on $C_{1}$ and the third on $C_{2}$. If $T p_{1}=T p_{2}$, then the three vertices of an equilateral triangle with unit side would lie upon the unit circle having $T p_{1}=T p_{2}$ as center, a contradiction.

Lemma 3. The distance $3^{1 / 2}$ is preserved.

Proof. If a pair of vertices of a rhombus with unit sides are separated by a distance $3^{1 / 2}$ they must, from Lemma 1 , be transformed into a single point or onto two points which are separated by a distance $3^{1 / 2}$. Lemma 2 implies that the distance $3^{1 / 2}$ is preserved.

That the distance 2 is preserved is seen by considering the regular hexagon with unit side. The consecutive vertices of such a figure must be transformed consecutively onto the vertices of a congruent figure. This becomes apparent when one considers the component overlapping rhombi. Therefore, two diametrically opposite vertices 
(which are separated by a distance 2) are transformed onto points separated by the same distance. The extension of such overlapping rhombi to establish the preservation of any integral distance is obvious.

We shall now show that all distances of the form $A / 2^{B}$, where $A$ and $B$ are integers, are preserved. By an obvious extension of the proof of Lemma 2 , it follows that if $A\left(3^{1 / 2}-1\right) \leqq d\left(p_{1}, p_{2}\right) \leqq A\left(3^{1 / 2}+1\right)$ then $T p_{1} \neq T p_{2}$. Consider the isosceles triangle with two sides of length $2 A$ and the third of length $A$. The vertices of this triangle are transformed onto the vertices of a congruent triangle. Thus, the system of two intersecting circles, each of which has one of the sides of length $2 A$ as a diameter, is transformed into a congruent system since a circle of radius $A$ is transformed into a congruent circle. The point, say $p_{1}$, of intersection of the two circles which is coincident with a vertex of the isosceles triangle is transformed into the corresponding point of the congruent image system. The other point, say $p_{2}$, of intersection (which is the midpoint of the side of length $A$ ) must be transformed into a point of intersection of the circles of the congruent image system. Since $A\left(3^{1 / 2}-1\right)<d\left(p_{1}, p_{2}\right)=(A / 2)(15)^{1 / 2}$ $<A\left(3^{1 / 2}+1\right)$, it follows that $T p_{1} \neq T p_{2}$. Thus, the midpoint of the side of length $A$ is transformed into the midpoint of the line joining the images of its end points, and the distance $A / 2$ is preserved. By extension of this argument, the distances $A / 2^{2}$ and hence $A / 2^{B}$ are preserved.

Since the points $A / 2^{B}$ are dense on the non-negative portion of the real line, there can be constructed, corresponding to arbitrary points $p$ and $q$ of the plane, a sequence of points $\left\{q_{i}\right\}$, with each $d\left(p, q_{i}\right)$ of the form $A / 2^{B}$, where $\lim _{i \rightarrow \infty} d\left(p, q_{i}\right)=d(p, q)$, and where $\lim _{i \rightarrow \infty} q_{i}=q$. For, if $q_{i}$ is sufficiently close to $q$, both $q_{i}$ and $q$ will lie on a circle of arbitrarily small radius $1 / 2^{B}$. It follows that $T q_{i}$ and $T q$ lie on a circle of the same radius, and therefore $\lim _{i \rightarrow \infty} T q_{i}=T q$. Also, $\lim _{i \rightarrow \infty} d\left(T p, T q_{i}\right)=d(p, q)$, since, for all $i, d\left(p, q_{i}\right)$ is preserved. Therefore, $d(T p, T q)=d(p, q)$, and hence $T$ preserves all distances.

4. Proof of theorem for $3 \leqq n<\infty$. Generalization of Lemmas 1, 2, and 3. That $T$ is single-valued may be shown by considering an $(n-1)$-dimensional unit-edged simplex in $n$-space. To the $n$ vertices of this figure there can be adjoined in only two ways an additional vertex in such a way as to form an $n$-dimensional unit-edged simplex. By analogous reasoning to that used in proving Lemma 1 , the assumption that some point has more than one image leads to the contradiction that the distance between the images of two unit-distant 
points is either 0 or $[2(n+1) / n]^{1 / 2}$ (i.e., 0 or twice the altitude of an $n$-dimensional unit-edged simplex).

Clearly, the vertices of a $k$-dimensional $(k \leqq n)$ unit-edged simplex in $n$-space are transformed by $T$ onto the vertices of a congruent simplex, and the points on an $n$-dimensional hypersphere of unit radius are transformed into a congruent hypersurface. As a generalization of Lemma 2 , if $[2(n+1) / n]^{1 / 2}-1 \leqq d\left(p_{1}, p_{2}\right) \leqq[2(n+1) / n]^{1 / 2}$ +1 , then $T p_{1} \neq T p_{2}$. For, otherwise, there would exist an $n$-dimensional unit-edged simplex in $n$-space whose vertices have images which both comprise the vertices of a congruent simplex and yet lie upon an $n$-dimensional hypersphere of unit radius, a contradiction. (The edge of an $n$-dimensional equi-edged simplex inscribed in an $n$-dimensional hypersphere of unit radius is easily shown to be $[2(n+1) / n]^{1 / 2}>1$.)

The distances $[2(n+1) / n]^{1 / 2},[2(n+1) / n], \cdots,[2(n+1) / n]^{j / 2}$, ... are preserved. As in the proof of Lemma 3, it follows from the above generalizations of Lemma 1 and Lemma 2 that the distance $[2(n+1) / n]^{1 / 2}$ is preserved. Replacing the unit distance by the distance $[2(n+1) / n]^{1 / 2}$, it then follows that $[2(n+1) / n]$ is preserved, and thence $[2(n+1) / n]^{3 / 2}$ is preserved, etc.

Lemma 4. A unit circle and its center are transformed into a unit circle and its center, respectively.

Proof. For simplicity of exposition, the case $n=3$ will be treated separately.

By the generalization of Lemma 3, any spherical surface of radius $(2 / 3) 6^{1 / 2}$ is transformed into a congruent surface. Two such spherical surfaces whose centers are separated by a distance $(2 / 3)(15)^{1 / 2}$ intersect in a unit circle. Since $(2 / 3) 6^{1 / 2}-1<(2 / 3)(15)^{1 / 2}<(2 / 3) 6^{1 / 2}+1$, it follows from the generalization of Lemma 2 that this unit circle is transformed into the intersection of two distinct spherical surfaces. Thus, any unit circle in 3-space is transformed into a circle.

Now consider the vertices of a regular hexagon $p_{1}, p_{2}, \cdots, p_{0}$ inscribed in a unit circle $C_{1}$. Since $T p_{i} \neq T p_{j}(i \neq j)$ by the generalization of Lemma 2 , and since $d\left(p_{1}, p_{2}\right)=d\left(p_{2}, p_{3}\right)=\cdots=d\left(p_{6}, p_{1}\right)=1$, it follows that $T p_{1}, T p_{2}, \cdots, T p_{6}$ on $T C_{1}$ are the vertices of a regular hexagon with unit side. Therefore, $C_{1}$ is transformed into a unit circle, and the image circle's center, which is the only point in space at unit distance from $T p_{1}, T p_{2}, \cdots, T p_{6}$, must be the image of the center of $C_{1}$.

The case $4 \leqq n<\infty$ will now be treated.

It will first be shown that, if $s$ is a preserved distance, then the 
surface of any 3-dimensional sphere of radius $s[(n-1) / 2(n-2)]^{1 / 2}$ in $n$-space is transformed into a congruent surface. Let coordinate axes in $n$-space be designated by $X_{1}, X_{2}, \cdots, X_{n}$. Consider an $(n-3)$ dimensional equi-edged simplex (edge $=s$ ) which is situated with its centroid at the origin, and in the intersection of hyperplanes $x_{1}=x_{2}$ $=x_{3}=0$. Let the vertices of this simplex be designated by $(0,0,0$, $\left.c_{4}^{(j)}, c_{5}^{(j)}, \cdots, c_{n}^{(j)}\right), j=1,2, \cdots, n-2$. Let $n-2 n$-dimensional hyperspheres of radius $s$ be located so that each of the vertices of the simplex is a center of one hypersphere. The equations of these hyperspherical surfaces are then: $x_{1}^{2}+x_{2}^{2}+x_{3}^{2}+\left(x_{4}-c_{4}^{(j)}\right)^{2}+\cdots+\left(x_{n}\right.$ $\left.-c_{n}{ }^{(j)}\right)^{2}=s^{2}, j=1,2, \cdots, n-2$. By subtraction, we obtain from these equations the equations: $\left(c_{4}^{(1)}-c_{4}^{(j)}\right) x_{4}+\cdots+\left(c_{n}^{(1)}-c_{n}^{(j)}\right) x_{n}=0$, $j=2,3, \cdots, n-2$. Since the determinant of the array of coefficients of this last set of equations is nonzero, it follows that $x_{4}=x_{5}=\ldots$ $=x_{n}=0$. Since $\sum_{t=4}^{n}\left(c_{t}^{(j)}\right)^{2}=(n-3) s^{2} / 2(n-2)$, the common intersection of the $n-2$ hyperspherical surfaces is expressed by the equations: $x_{1}^{2}+x_{2}^{2}+x_{3}^{2}=(n-1) s^{2} / 2(n-2)$ and $x_{4}=x_{5}=\cdots=x_{n}=0$. Thus, the surface of any 3 -dimensional sphere of radius $s[(n-1) / 2(n-2)]^{1 / 2}$ can be considered to be the intersection of such a system of $n-2$ hyperspherical surfaces of radius $s$, when the origin of the coordinate system is taken at the center of the 3-dimensional sphere. The image of this system of hyperspherical surfaces lies in a congruent system, and therefore the surface of the 3-dimensional sphere is transformed into the surface of a congruent 3-dimensional sphere.

It will now be shown that any unit circle in $n$-space is transformed into a circle. Any unit circle can be considered to be the intersection of the surfaces of two 3-dimensional spheres of radii $[2(n+1) / n]^{1 / 2}$ . $[(n-1) / 2(n-2)]^{1 / 2}$ and $[2(n+1) / n][(n-1) / 2(n-2)]^{1 / 2}$. By the preceding paragraph, these spherical surfaces are transformed into surfaces which are congruent to them. These, consequently distinct, spherical image surfaces contain the image of the unit circle in their circle of intersection.

The completion of the proof of Lemma 4 for $4 \leqq n<\infty$ follows by the second paragraph of the argument for the case $n=3$.

\section{Lemma 5. A plane is transformed into a plane.}

Proof. Let $C_{1}$ be a unit circle in $n$-space with center $o_{1}$, and let $p$ be an arbitrary point in the plane of $C_{1}$ such that $d\left(p, o_{1}\right) \leqq 2$. Construct a co-planar unit circle, $C_{2}$, passing through $p$ with its center on the circumference of $C_{1}$. By Lemma $4, C_{1}$ and $C_{2}$ are transformed into intersecting unit circles. Furthermore, the images of the two points of intersection of the circles $C_{1}$ and $C_{2}$ and the image of $o_{1}$ can be seen 
to determine the plane of both $T C_{1}$ and $T C_{2}$. Therefore, $T p$ lies in this plane and all points in the plane of $C_{1}$ within a distance 2 from $o_{1}$ are transformed into co-planar points. Obviously, this region can be extended in the plane of $C_{1}$ indefinitely, since $C_{1}$ could now, in the above argument, be chosen to be a unit circle which is internally tangent to the boundary of this region.

Since planes are transformed into planes, it follows, from the proof of the theorem for the case $n=2$, that all distances are preserved.

International Business Machines Corporation 